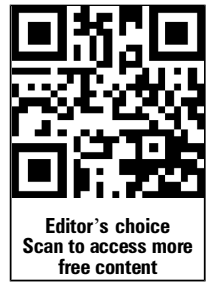

- Additional material is published online only. To view please visit the journal online (http://dx.doi.org/10.1136/ heartjnl-2013-304960).

${ }^{1}$ Section of Epidemiology and Biostatistics, University of Auckland, Auckland, New Zealand

${ }^{2}$ Department of Planning and Funding, Waitemata District Health Board, Auckland, New Zealand

${ }^{3}$ School of Pharmacy, University of Auckland, Auckland, New Zealand ${ }^{4}$ Middlemore Hospital, Auckland, New Zealand

\section{Correspondence to} Dr Corina Grey, Section of Epidemiology and Biostatistics, School of Population Health, University of Auckland, PO Box 92019, Auckland 1142, New Zealand; c.grey@auckland.ac.nz

Received 12 September 2013 Revised 19 December 2013 Accepted 23 December 2013 Published Online First 16 January 2014

\title{
Maintenance of statin use over 3 years following acute coronary syndromes: a national data linkage study (ANZACS-QI-2)
}

\author{
Corina Grey, ${ }^{1}$ Rod Jackson, ${ }^{1}$ Sue Wells, ${ }^{1}$ Simon Thornley, ${ }^{1}$ Roger Marshall, ${ }^{1}$ \\ Sue Crengle, ${ }^{2}$ Jeff Harrison, ${ }^{3}$ Tania Riddell, ${ }^{1}$ Andrew Kerr ${ }^{1,4}$
}

\section{ABSTRACT}

Objective To describe patterns of statin use and predictors of poor maintenance over a 3-year period following an acute coronary syndrome (ACS).

Methods National hospitalisation, mortality and pharmaceutical dispensing data were linked for all subjects aged 35-84 years discharged from a public hospital with an ACS in New Zealand in 2007. A Medication Possession Ratio (MPR; percentage of follow-up days patients were dispensed statins) was calculated for each patient. Adequate maintenance was defined by a MPR $\geq 80 \%$.

Results In 2007, 11348 patients aged 35-84 years were discharged from hospital with ACS. Within 90 days of discharge, $83 \%$ had received a statin. Over the follow-up period, $66 \%$ were adequately maintained on a statin (MPR $\geq 80 \%$ ): $69 \%$ in the first year, $67 \%$ in the second year and $66 \%$ in the third year. Patients taking statins prior to admission and those who underwent a coronary procedure were $20-50 \%$ more likely to have a MPR $\geq 80 \%$ over 3 years than others. In contrast, people aged $35-45$ years and those of Maori or Pacific ethnicity were $13-25 \%$ less likely to have a MPR $\geq 80 \%$ than those aged 55-64 years and Europeans.

Conclusions One-third of patients were not adequately maintained on statins over the 3-year period following ACS, but $82 \%$ of those on a statin prior to admission had an MPR $\geq 80 \%$ over 3 years of follow-up. These findings define achievable treatment levels and identify groups who may benefit from efforts to improve statin use.

\section{INTRODUCTION}

Despite the well-documented safety and effectiveness of statins for the secondary prevention of cardiovascular disease (CVD), ${ }^{1}{ }^{2}$ many studies report suboptimal statin use in people following an acute coronary syndrome (ACS). Among patients dispensed statins at least once after an ACS, studies suggest that one-half to three-quarters will have discontinued their medications by 2 years after discharge. $^{3-5}$

In New Zealand there are few external barriers to long-term statin use. The universal healthcare system has a strong primary care focus, in-hospital medications are free of charge and statins are heavily subsidised by the government (at the time of the study, each 3-month prescription cost NZD\$3). The country also has comprehensive up-to-date data on public hospitalisations, mortality and government-subsidised pharmaceutical dispensing. Using these linked datasets, we have previously investigated 1-year statin maintenance among patients hospitalised for acute coronary events. ${ }^{6}$ In this study we assessed statin maintenance for up to 3 years and identified independent predictors of statin use among patients with ACS.

\section{METHODS}

\section{Study population}

We included all New Zealand residents aged 3584 years discharged from a public hospital with a diagnosis of ACS (ST elevation myocardial infarction (STEMI), non-ST elevation MI (NSTEMI), MI unspecified and unstable angina (UA)) between 1 January and 31 December 2007. The ICD-10-AM codes used to define the four ACS types used in the analysis are listed in online supplementary appendix 1 . We excluded people who died within 90 days of discharge or those who spent less than 90 days out of hospital over follow-up as statin use in this subset is likely to differ significantly from other patients. We also excluded patients aged $<35$ years due to diagnostic uncertainty and those aged $\geq 85$ years as treatment decisions for this group are likely to be significantly influenced by factors such as quality of life and comorbidities. While comorbidities may also be common in patients aged 75-84 years, they represent about one-third of the total ACS population and are therefore an important group to investigate.

\section{Data sources}

The study cohort was created by linking national datasets of public hospital diagnoses, mortality records and national pharmaceutical claims data. Linkage was carried out using an encrypted version of the National Health Index number, a unique identifier assigned to all New Zealand residents who have had contact with publicly-funded health services (about $98 \%$ of the population). ${ }^{7}$ Private hospital admissions were excluded because data are unreliable and represent $<5 \%$ of all coronary heart disease (CHD) hospitalisations, mostly chronic $\mathrm{CHD}$ and non-acute procedures. ${ }^{8}$

\section{Main outcome measure}

Statin use over a 3-year follow-up period (starting at the discharge date of the person's index event and ending 1095 days later) was assessed by calculating a Medication Possession Ratio (MPR) for each person. The MPR was the number of days a 
statin was assumed to be in a patient's possession (based on dispensed drugs) divided by the number of days spent out of hospital from the date of hospital discharge through to the end of the follow-up period or the date of death, whichever came first. Days spent in hospital were excluded because medications supplied to inpatients are not included in the national drug dispensing database. For patients dispensed a statin in the 90 days before hospitalisation, the number of statin days remaining at the time of their hospitalisation was added to the numerator of the MPR; therefore, some patients had a MPR >1.0.

The possession of statins during $80 \%$ or more of follow-up time (ie, MPR $\geq 0.8$ ) was used to classify those adequately maintained on medications, as reported elsewhere. ${ }^{9}$ We also considered $\mathrm{MPR}=0$, indicating no dispensing at all. The MPR is a direct measure of medication dispensing and is assumed to be a reasonable proxy measure of medication use.

An overall MPR was calculated for the entire cohort and subgroups of it, regardless of death during follow-up, based on total days of medication supplied and total days of follow-up spent in the community. MPRs were also calculated separately for each year of follow-up. MPRs for the second and third years excluded deaths in previous years.

\section{Other variables}

Socioeconomic deprivation was assessed by the NZDep01 score, a census-based small-area 10-point index of deprivation based on a person's domicile. ${ }^{10}$ This score was aggregated into three groups: $1-3$ the least deprived, $8-10$ the most deprived and 4-7 intermediate. Ethnicity was determined from hospital records and, if more than one ethnicity was recorded, it was prioritised in the following order: Māori, Pacific, Indian and New Zealand European/other; so, for example, a person identified as both European and Māori is classified as Māori. A coronary procedure was considered to have occurred during a patient's index hospitalisation if it was performed within 30 days of the event.

Diabetes was determined from previous hospitalisations and/or pharmaceutical dispensing data. ${ }^{11}$ A history of CVD was defined by hospitalisation between 2002 and the index event with any of the following conditions: $\mathrm{CHD}$, a coronary procedure, heart failure, ischaemic stroke, atherosclerotic peripheral vascular disease and/or a peripheral arterial procedure (see online supplementary appendix 1 for ICD-10-AM codes). Hospitalisation data prior to 2002 were not used as they are incomplete.

\section{Statistical analysis}

Descriptive statistics were used to compare patient characteristics among ACS groups. Poisson regression modelling using a robust variance estimator was used to identify predictors of statin maintenance after 1 year and up to 3 years of follow-up and to estimate relative risks for adequate statin maintenance. $^{12} 13$ Variables considered in the modelling included: ACS type, age group, gender, ethnicity, socioeconomic deprivation, statin use in the 90 days prior to admission, coronary procedure, history of CVD, diabetes, time spent in hospital and interaction of ACS type with age, gender and ethnicity, respectively. Variables were considered significantly associated with statin use when $\mathrm{p}<0.05$. Analyses were conducted in Stata statistical software V.11.0. ${ }^{14}$

\section{RESULTS}

Between 1 January and 31 December 2007, 16591 people were discharged from a public hospital with ACS. Applying exclusion criteria, 631 were not New Zealand residents and 3097 were excluded because 55 were aged $<35$ years and 3042 were aged $\geq 85$ years. A further 1429 died within 90 days of discharge and another 86 spent less than 90 days out of hospital over the follow-up period. A total of 3371 people (26\%) who met age and residency inclusion criteria died during the 3 years of follow-up with over $40 \%$ of these deaths occurring in the first 90 days.

Once exclusions were applied, 11348 people remained in the study cohort. Baseline characteristics, stratified by ACS type, are shown in table 1 . Approximately half the cohort was diagnosed with NSTEMI, 34\% with UA, 14\% with STEMI and 3\% with unspecified MI. Compared with other ACS types, the group with STEMI was younger, had fewer women and fewer subjects with a history of CVD, diabetes and prior statin use, but more who had undergone a coronary procedure.

Table 1 Characteristics of total study cohort and by ACS type

\begin{tabular}{|c|c|c|c|c|c|}
\hline n (\% of total) & $\begin{array}{l}\text { Total cohort } \\
\mathrm{N}=11348\end{array}$ & $\begin{array}{l}\text { STEMI } \\
\mathrm{n}=1555\end{array}$ & $\begin{array}{l}\text { NSTEMI } \\
\mathrm{n}=5611\end{array}$ & $\begin{array}{l}\text { MI unspecified } \\
n=310\end{array}$ & $\begin{array}{l}\text { Unstable angina } \\
\mathrm{n}=3872\end{array}$ \\
\hline Mean age (SD) & $67.2(11.7)$ & $63.0(12.2)$ & $68.3(11.7)$ & $67.6(11.5)$ & $67.4(11.1)$ \\
\hline Women & $4198(37 \%)$ & $423(27 \%)$ & $2135(38 \%)$ & $121(39 \%)$ & 1519 (39\%) \\
\hline European/other & $9538(84 \%)$ & $1310(84 \%)$ & $4667(83 \%)$ & $253(82 \%)$ & 3308 (85\%) \\
\hline Māori & $1168(10 \%)$ & $144(9 \%)$ & $604(11 \%)$ & $39(13 \%)$ & $381(10 \%)$ \\
\hline Pacific & $391(3 \%)$ & $54(4 \%)$ & $231(4 \%)$ & $10(3 \%)$ & $96(3 \%)$ \\
\hline Indian & $251(2 \%)$ & $47(3 \%)$ & $109(2 \%)$ & $8(3 \%)$ & $87(2 \%)$ \\
\hline Least deprived 3 deciles* & $2269(20 \%)$ & $323(21 \%)$ & $1153(21 \%)$ & $54(17 \%)$ & $739(19 \%)$ \\
\hline Intermediate 4 deciles & $4739(42 \%)$ & $657(42 \%)$ & $2330(42 \%)$ & $132(43 \%)$ & $1620(42 \%)$ \\
\hline Most deprived 3 deciles & $4307(38 \%)$ & $568(37 \%)$ & $2111(38 \%)$ & $124(40 \%)$ & $1505(39 \%)$ \\
\hline Prior statint & $4964(44 \%)$ & $357(23 \%)$ & 2205 (39\%) & $103(33 \%)$ & 2299 (59\%) \\
\hline Diabetes & $3263(29 \%)$ & $307(20 \%)$ & $1723(31 \%)$ & $87(28 \%)$ & $1146(30 \%)$ \\
\hline History of CVD & $3876(34 \%)$ & $232(15 \%)$ & $1796(32 \%)$ & $87(28 \%)$ & $1761(45 \%)$ \\
\hline Coronary procedure $\ddagger$ & $3724(33 \%)$ & $884(57 \%)$ & $1753(31 \%)$ & $93(30 \%)$ & $994(26 \%)$ \\
\hline
\end{tabular}

*NZDep01 data were missing in 33 people ( $<1 \%$ of cohort).

†Statin dispensed in the 90 -day period prior to index admission.

¥Angioplasty or coronary bypass grafting within 30 days of index admission.

ACS, acute coronary syndrome; CVD, cardiovascular disease; STEMI, ST elevation myocardial infarction; NSTEMI, non-ST elevation myocardial infarction. 
Table 2 Proportion of people with a Medication Possession Ratio (MPR) $\geq 0.8$ during follow-up

\begin{tabular}{|c|c|c|c|}
\hline & & Total cohort & $\begin{array}{l}\text { Patients still alive at } \\
\text { the end of follow-up ( } n=9490)\end{array}$ \\
\hline Time period & Number included in analysis* & $\%$ with $M P R \geq 0.8 \dagger$ & $\%$ with MPR $\geq 0.8 \dagger$ \\
\hline Year 1 & 11348 & 69 & 71 \\
\hline Year 2 & 10645 & 67 & 68 \\
\hline Year 3 & 9988 & 66 & 66 \\
\hline Years 1-3 & 11348 & 66 & 68 \\
\hline
\end{tabular}

*To account for deaths during follow-up, this analysis also excluded patients who did not spend any days out of hospital during that year.

tDuring the time period stated in the left-hand column of the table.

Overall, 59\% of the cohort had a statin dispensed within 7 days of hospital discharge, $71 \%$ within 30 days and $83 \%$ within 90 days.

\section{Statin use over 3 years}

Using a MPR threshold of $0.8,66 \%$ of the cohort were adequately treated with a statin up to 3 years after hospital discharge (table 2). Overall, statin use remained relatively stable, with $69 \%$ of the cohort achieving a MPR $\geq 0.8$ in the first year, $67 \%$ in the second year and $66 \%$ in the third year; $10 \%$ of patients received no statins during the 3 -year follow-up period.

In patients still alive at the end of follow-up $(n=9490)$, we examined changes in maintenance over the study period. Of those who received no statin in the first year $(\mathrm{MPR}=0), 82 \%$ received none in the third year. Similarly, $82 \%$ of those who were adequately maintained on a statin in the first year (MPR $\geq 0.8$ ) were also adequately maintained in year 3 . However, $4 \%$ of patients with optimal dispensing in the first year received no statin in the third year and $14 \%$ had inadequate statin maintenance.

Online supplementary figures S1-7 in appendix 2 show how the proportion of people with a MPR $\geq 0.8$ varied between different levels of demographic and clinical subgroups.

Table 3 Adjusted* relative risk $(95 \% \mathrm{Cl})$ of maintaining a Medication Possession Ratio $\geq 0.8$ over 1 year and up to 3 years following an ACS hospitalisation

\begin{tabular}{lll}
\hline & 1 year & Up to 3 years \\
\hline $\begin{array}{l}\text { Age } 35-44 \text { years } \\
\text { Age 45-54 years }\end{array}$ & $0.84(0.77$ to 0.91$)$ & $0.75(0.67$ to 0.83$)$ \\
Age 55-64 years & $0.97(0.93$ to 1.01$)$ & $0.94(0.90$ to 0.99$)$ \\
Age $65-74$ years & $1.00(0.97$ to 1.03$)$ & $1.02(0.99$ to 1.06$)$ \\
Age $75-84$ years & $0.96(0.93$ to 1.00$)$ & $0.97(0.94$ to 1.01$)$ \\
Female & $0.95(0.93$ to 0.98$)$ & $0.94(0.91$ to 0.96$)$ \\
European/other & & \\
Māori & $0.88(0.84$ to 0.92$)$ & $0.87(0.83$ to 0.92$)$ \\
Pacific & $0.82(0.76$ to 0.89$)$ & $0.82(0.75$ to 0.90$)$ \\
Indian & $1.01(0.94$ to 1.08$)$ & $0.98(0.91$ to 1.07$)$ \\
STEMI & $1.18(1.14$ to 1.22$)$ & $1.15(1.10$ to 1.19$)$ \\
Coronary procedure & $1.24(1.21$ to 1.27$)$ & $1.22(1.19$ to 1.25$)$ \\
Previous CVD & $0.94(0.91$ to 0.96$)$ & $0.96(0.93$ to 0.98$)$ \\
Prior statin use & $1.57(1.53$ to 1.62$)$ & $1.56(1.52$ to 1.61$)$
\end{tabular}

All figures in parenthesis are $95 \% \mathrm{Cls}$.

*Adjusted for the variables shown in this table: age, gender, ethnicity, STEMI diagnosis, coronary procedure within 30 days of admission, previous CVD and statin use in the 90 days before admission.

ACS, acute coronary syndrome; CVD, cardiovascular disease; STEMI, ST elevation myocardial infarction.

\section{Multivariate analysis}

The relative risks of being adequately maintained on a statin over 1 and 3 years, adjusted for clinical and demographic factors, are shown in table 3 . A relative risk of $>1$ is associated with better statin maintenance.

Socioeconomic deprivation and time spent in hospital were not statistically significant and were not used as adjustment variables. STEMI was included in the models as an adjustment variable rather than the four classes of ACS type, as statin maintenance in this group of patients differed markedly from other groups. There was no evidence for an interaction between STEMI and ethnicity, age and gender ( $p>0.3$ for each).

After adjusting for all other variables, being young $(<45$ years) and of Maori or Pacific ethnicity were the strongest predictors of poor statin maintenance. The strongest predictors of good statin maintenance were previous statin use, having a coronary procedure and a diagnosis of STEMI. Women and those with a history of CVD were slightly less likely to be maintained on a statin than men and those without a history of CVD, respectively. Similar patterns were also observed for statin maintenance over 1 year of follow-up.

Models were also constructed to include only survivors of the entire 3-year follow-up period. These yielded similar results to those presented, indicating that the factors associated with statin maintenance and their magnitude of effect were the same regardless of death during follow-up (data not shown).

\section{DISCUSSION}

In this national cohort of people discharged from hospital following an ACS in 2007, 83\% were dispensed statins within 90 days of discharge and $66 \%$ maintained an adequate supply of medication overall with minimal change in the second and third years. Younger people and those of Maori and Pacific ethnicity had poorer statin maintenance. Over the 3 years of follow-up, only $46 \%$ of people aged $35-44$ years, $54 \%$ of Pacific peoples and 55\% of Maori were adequately maintained on a statin compared with $71 \%$ of people aged $65-74$ years, $68 \%$ of European/others and $71 \%$ of Indians. Of those on a statin prior to admission, $82 \%$ were adequately maintained on a statin over the follow-up period.

Rates of statin maintenance in our study are higher than reported in previous studies from New Zealand $59 \%$ over 1 year), ${ }^{6}$ Canada ( $40 \%$ over 2 years) ${ }^{15}$ and the USA (43\% over 3 years). ${ }^{16}$ However, a higher rate was reported in France $(76 \%$ over 30 months) using data from the general health insurance scheme, which covered $70 \%$ of the population and included only those with STEMI or NSTEMI. ${ }^{17}$ The lower statin dispensing reported in the other studies may be due in part to their inclusion of patients with angina ${ }^{6}$ and data that are now more than 10 years old. ${ }^{15}$ 
One of the strengths of our study is that almost all patients with ACS aged 35-84 years in New Zealand during the study period were included. Also, as all statins are heavily publicly subsidised, it is likely that nearly all statin dispensing in the community is captured in the national pharmaceutical database. Another strength is the length of follow-up. Internationally, few studies have examined statin use past 1 year post-ACS. ${ }^{18}$ Moreover, studies with longer follow-up tend to be small, ${ }^{16}$ use data more than 10 years old ${ }^{19}$ or samples that may not be representative of the entire population. ${ }^{420}$

Individual patterns of statin maintenance in our cohort changed a little between the first and third years after discharge. We found that $82 \%$ of patients who maintained an optimal statin MPR in the first year continued to do so in the third year. Of the remaining $18 \%$ of patients, $4 \%$ stopped statins completely. This suggests that, if adequate statin maintenance can be achieved in the first year following an ACS, patients are likely to continue it subsequently.

Our finding that previous statin use was a predictor of subsequent use is consistent with previous studies. ${ }^{6}{ }^{21}$ It is likely that many of those on statins prior to admission are long-term statin users. As most statin intolerance occurs early, these long-term users are less likely than newly prescribed patients to discontinue due to intolerance; they have also demonstrated adherence through previous dispensings. In contrast, patients with a prior indication but no recent statin dispensing are more likely to have had a real or perceived statin intolerance or other reasons for nonadherence. One strategy to address these issues is discharge counselling, ${ }^{5}$ where any patient with CVD not already taking statins receives specific therapeutic advice. This would need to be delivered within a multimodal approach. ${ }^{22}$ In particular, those with previous side effects should be carefully evaluated as the label of 'statin intolerant' is difficult to change after discharge. Our experience is that it is often possible to successfully establish such patients on an alternative statin or a reduced dose.

As mentioned above, young people and those of Māori or Pacific ethnicity have poor statin maintenance. The finding concerning age is consistent with some studies, ${ }^{20} 2{ }^{24}$ but not others. $^{5} 15 \quad 25$ The effect of ethnicity has not been welldescribed, with one meta-analysis identifying only three out of 22 eligible papers that examined ethnicity as a predictor of statin maintenance. ${ }^{21}$ All three studies found that non-white ethnic groups were less likely to be maintained on statins than white patients. $^{2325} 26$ The reasons for these ethnic differences are likely to be multifactorial and may be related to differences in prescribing patterns for people of different ethnic groups, language and/or cultural barriers that may lead to differential access to healthcare or health behaviours. ${ }^{27}$

Our results suggest that targeted interventions to increase the use of statin therapy after ACS will be necessary, particularly for young people and some ethnic groups. Younger patients with ACS are at the highest lifetime risk of CHD, but may be less likely to perceive themselves as being at risk. ${ }^{21}$ They stand to gain most from secondary prevention therapy: life expectancy is approximately halved in patients with a history of MI at all ages, but absolute differences are much greater in younger than in older individuals. ${ }^{28}$ There are marked ethnic inequalities in cardiovascular health in New Zealand, ${ }^{29}$ so it is vital that every effort is made to increase the use of effective preventive treatments in Māori and Pacific peoples.

An important limitation of studies like ours, based on routine datasets, is the lack of clinical information on comorbidities other than prior CVD and diabetes or on common risk factors. There were no data on medication prescribing (only dispensing) or whether patients had a history of statin intolerance. Therefore, we cannot be certain what proportion of the calculated statin underuse was due to underprescribing, patients' decisions not to take medications, the occurrence of side effects or other contraindications. Second, we used dispensing of statins as a marker of statin use, but some patients may not have taken their medications. However, this method is considered to have high specificity (identify those not taking medication) and is more likely than clinical trials to reflect a 'real world' situation. ${ }^{9}$ Third, the proportion of people with a history of CVD is likely to be underestimated as hospitalisation data prior to 2002 were not included.

In conclusion, this national study has demonstrated that one-third of patients with ACS are not adequately maintained on a statin 3 years after discharge, and that previous statin use, age and ethnicity are important predictors of future statin use. The significant differences we observed indicate that long-term maintenance levels of $>80 \%$ are achievable but are only $50 \%$ in some groups. Knowing which patients may be at increased risk of discontinuation of this cheap and effective therapy could help healthcare organisations to develop better support and follow-up systems for these high-risk patient groups.

\section{Key messages}

What is already known on this subject

- Long-term statin use is well-established as a safe and effective component of secondary prevention following an acute coronary syndrome (ACS). Studies suggest that stain use is suboptimal even over the short term, and that increasing age and previous statin use may be important predictors of statin maintenance following an ACS.

\section{What this study adds}

- We examined statin use over a longer time period than most previous studies and found that $66 \%$ of patients with ACS were adequately maintained on a statin up to 3 years after their original coronary event. Of patients dispensed optimal amounts of statin in the first year, $82 \%$ were still taking a statin in the third year. People of younger age and/or of Maori or Pacific ethnicity were less likely, whereas those who had a coronary procedure were more likely, to be maintained on statin treatment over the long term.

\section{How this might impact on clinical practice}

- Our findings suggest that, if adequate statin maintenance can be achieved in the first year following an ACS, a patient is likely to continue it in subsequent years. Efforts to increase statin use need to consider the particular needs of the young and those of Maori or Pacific ethnicity.

Acknowledgements Our thanks to the National Health Board Analytic Services and Pharmac for enabling use of these data.

Contributors CG, RJ, SW, ST and AK were involved in the design of the study. CG analysed the data. CG, RJ, SW and AK interpreted the data. CG drafted the manuscript and all authors were responsible for critically reviewing it and approving the final submission.

Funding This research project has been supported by the Health Research Council (grant number 11/800). CG and ST are supported by Health Research Council Clinical Research Training Fellowships (grant numbers $11 / 145$ and 11/814). SW is partly funded by the Stephenson Foundation. 
Competing interests None.

Ethics approval Ethics approval was obtained from the Multiregion Ethics Committee.

Provenance and peer review Not commissioned; externally peer reviewed.

\section{REFERENCES}

1 Scandinavian Simvastatin Survival Study Group. Randomized trial of cholesterol lowering in 4444 patients with coronary heart disease: the Scandinavian Simvastatin Survival Study (4S). Lancet 1994;344:1383-9.

2 Sacks FM, Pfeffer MA, Moye LA, et al. The effect of pravastatin on coronary events after myocardial infarction in patients with average cholesterol levels. N Engl J Med 1996:339:1349-57.

3 Choudhry NK, Setoguchi S, Levin R, et al. Trends in adherence to secondary prevention medications in elderly postmyocardial infarction patients. Pharmacoepidemiol Drug Saf 2008;17:1189-96.

4 Chodick G, Shlev V, Gerber Y, et al. Long-term persistence with statin treatment in a not-for-profit health maintenance organization: a population-based retrospective cohort study in Israel. Clin Ther 2008;30:2167-79.

5 Jackevicius CA, Li P, Tu JV. Prevalence, predictors, and outcomes of primary nonadherence after acute myocardial infarction. Circulation 2008;117:1028-36.

6 Thornley S, Marshall R, Chan WC, et al. Four out of ten patients are not taking statins regularly during the 12 months after an acute coronary event. Eur J Prev Cardiol 2012:19:349-57.

7 Ministry of Health. National Health Index data dictionary (version 5.3). Ministry of Health, 2009.

8 Chan WC, Wright C, Tobias M, et al. Explaining trends in coronary heart disease hospitalisations in New Zealand: trend for admissions and incidence can be in opposite directions. Heart 2008;94:1589-93.

9 Andrade SE, Kahler KH, Frech F, et al. Methods for evaluation of medication adherence and persistence using automated databases. Pharmacoepidemiol Drug Saf 2006;15:565-74.

10 Salmond C, Crampton P. NZ Dep 2001 Index of Deprivation. User's Manual. Wellington: Wellington School of Medicine and Health Sciences, 2002.

11 Thornley S, Wright C, Marshall R, et al. Can the prevalence of diagnosed diabetes be estimated from linked national health records? The validity of a method applied in New Zealand. J Prim Health Care 2011;3:262-8.

12 Barros AJD, Hirakata VN. Alternatives for logistic regression in cross-sectional studies: an empirical comparison of models that directly estimate the prevalence ratio. BMC Med Res Methodol 2003;3:21

13 Zou G. A modified Poisson regression approach to prospective studies with binary data. Am J Epidemiol 2004;159:702-6.
14 StataCorp. Stata Statistical Software: Release 11. College Station, TX: StataCorp LP, 2009.

15 Jackevicius CA, Mamdani M, Tu JV. Adherence with statin therapy in elderly patients with and without acute coronary syndromes. JAMA 2002;288:462-7.

16 Shah ND, Dunlay SM, Ting HH, et al. Long-term medication adherence after myocardial infarction: experience of a community. Am J Med 2009; 122:961.e7-13.

17 Tuppin P, Neumann A, Danchin N, et al. Evidence-based pharmacotherapy after myocardial infarction in France: adherence-associated factors and relationship with 30-month mortality and rehospitalization. Arch Cardiovasc Dis 2010; 103:363-75.

18 Kirchmayer U, Agabitis N, Belleudi V, et al. Socio-demographic differences in adherence to evidence-based drug therapy after discharge from acute myocardial infarction: a population-based cohort study in Rome, Italy. J Clin Pharm Ther 2012;37:37-44

19 Gislason GH, Rasmussen JN, Abildstrom SZ, et al. Long-term compliance with beta-blockers, angiotensin-converting enzyme inhibitors, and statins after acute myocardial infarction. Eur Heart J 2006;27:1153-8.

20 Hudson M, Richard H, Pilote L. Parabolas of medication use and discontinuation after myocardial infarction-are we closing the treatment gap? Pharmacoepidemiol Drug Saf 2007;16:773-85.

21 Mann DM, Woodward M, Muntner $P$, et al. Predictors of nonadherence to statins: a systematic review and meta-analysis. Ann Pharmacother 2010;44:1410-21.

22 Haynes RB, Ackloo E, Sahota N, et al. Interventions for enhancing medication adherence. Cochrane Database Syst Rev 2008;(2):CD000011.

23 Ellis JJ, Erickson SR, Stevenson JG, et al. Suboptimal statin adherence and discontinuation in primary and secondary prevention populations. J Gen Intern Med 2004; 19:638-45

24 Esposti LD, Saragoni S, Batacchi P, et al. Adherence to statin treatment and health outcomes in an Italian cohort of newly treated patients: results from an administrative database analysis. Clin Ther 2012;34:190-9.

25 Benner J, Glynn R, Mogun H, et al. Long-term persistence in use of statin therapy in elderly patients. JAMA 2002;288:455-61.

26 Mann DM, Allegranate JP, Natarajan S, et al. Predictors of adherence to statins for primary prevention. Cardiovasc Drugs Ther 2007;21:311-16.

27 Tukuitonga C, Bindman A. Ethnic and gender differences in the use of coronary artery revascularisation procedures in New Zealand. N Z Med J 2002;115.

28 Peeters A, Manmun AA, Willekens F, et al. A cardiovascular life history - a life course analysis of the original Framingham Heart Study cohort. Eur Heart $J$ 2002;23:458-66.

29 Bramley $\mathrm{D}$, Hebert $\mathrm{P}$, Jackson $\mathrm{R}$, et al. Indigenous disparities in disease-specific mortality, a cross-country comparison: New Zealand, Australia, Canada and the United States. N Z Med J 2004;117:1207. 